\title{
XTEN-Annexin A5: XTEN Allows Complete Expression of Long-Circulating Protein-Based Imaging Probes as Recombinant Alternative to PEGylation
}

\author{
Akvile Haeckel*1, Franziska Appler*1, Lena Figge ${ }^{1}$, Harald Kratz ${ }^{1}$, Mathias Lukas ${ }^{2}$, Roger Michel ${ }^{2}$, Jörg Schnorr ${ }^{1}$, \\ Marietta Zille ${ }^{3}$, Bernd Hamm ${ }^{1}$, and Eyk Schellenberger ${ }^{1}$ \\ ${ }^{I}$ Institute of Radiology—Molecular Imaging Group, Charité, Berlin, Germany; ${ }^{2}$ Clinic of Nuclear Medicine, Charité, Berlin, \\ Germany; and ${ }^{3}$ Clinic of Neurology—Department of Experimental Neurology, Charité, Berlin, Germany
}

The coupling of polyethylene glycol (PEG) to proteins (PEGylation) has become a standard method to prolong blood circulation of imaging probes and other proteins, liposomes, and nanoparticles. However, concerns have arisen about the safety of PEG, especially with respect to its poor biodegradability and antibody formation, including new evidence about preformed anti-PEG antibodies in a quarter of healthy blood donors. Here, we apply a new hydrophilic polypeptide XTEN to extend the blood half-life of an imaging probe. As an example, we chose annexin A5 (AnxA5), a recombinant $35-\mathrm{kD}$ protein extensively used for the in vitro and in vivo detection of apoptosis, that has a blood half-life of less than $7 \mathrm{~min}$ in mice, limiting its accumulation in target tissues and therefore limiting its utility as an imaging reagent. Methods: The sequence of XTEN was developed by Volker Schellenberger and colleagues by evolutionary in vitro optimization to yield PEG-like properties but provides several key advantages in comparison to PEG. The DNA of a 288-amino-acid version of XTEN with an additional Nterminal cysteine for site-directed coupling was fused to AnXA5 (XTENAnxA5). The fusion protein could be highly expressed in Escherichia coli and efficiently purified using XTEN conveniently as a purification tag. It was labeled with a thiol-reactive fluorescent dye and via a chelator with a radionuclide. Results: SPECT/CT imaging revealed a blood half-life of about $1 \mathrm{~h}$ in mice, markedly longer than the 7-min blood half-life for unmodified AnxA5, which should allow improved imaging of target tissues with low perfusion. In comparison to AnxA5, XTENAnxA5 demonstrated a substantially higher accumulation in tumors under chemotherapy in near-infrared fluorescence imaging. Conclusion: The presented method allows the expression and production of high amounts of long-circulating XTEN-AnxA5 without the necessity of PEGylation, thereby simplifying the synthesis while avoiding labelinginduced inactivation of AnxA5 and potential adverse effects of PEG. It is readily applicable to other recombinant protein or peptide-based imaging probes and allows fine-tuning of the desired blood half-life, because longer XTEN variants yield longer blood half-lives.

Key Words: XTEN; polyethylene glycol; annexin A5; longcirculating; recombinant protein

J Nucl Med 2014; 55:508-514

DOI: 10.2967/jnumed.113.128108

Received Nov. 5, 2013; revision accepted Nov. 6, 2013.

For correspondence or reprints contact: Eyk Schellenberger, Institute of

Radiology, Charité, Charitéplatz 1, Berlin, 10117, Germany.

E-mail: eyk.schellenberger@charite.de

${ }^{*}$ Contributed equally to this work.

Published online Feb. 17, 2014.

COPYRIGHT (C) 2014 by the Society of Nuclear Medicine and Molecular Imaging, Inc.
$\mathbf{T}$ he covalent coupling of polyethylene glycol (PEG), so-called PEGylation, has been developed into a widely used technique to prolong the half-life of proteins, peptides, liposomes, and nanoparticles in the bloodstream. Commercial sources provide branched and linear PEGs in numerous sizes with various reactive linker groups. Because of the large hydrate shell of PEG, PEGylation can reduce the immunogenicity and toxicity of the substances to which it is coupled and decrease their clearance by renal filtration by exceeding the molecular size cutoff of the kidneys and preventing their degradation through proteolytic enzymes and macrophages $(1,2)$.

However, PEGylation has several disadvantages that are summarized in Table 1: one major problem is that PEG is virtually not biodegradable. Therefore, protein conjugates using PEG below the kidney filtration cutoff (e.g., PEG with $5 \mathrm{kDa}$ ) are preferred and considered safe if they are completely excreted. Although PEG has very low immunogenicity and is considered nontoxic, some animal studies have demonstrated that PEGylated proteins cause renal vacuolation in mice (3) and antibody production against PEG in mice $(4,5)$ and dogs (6). Moreover, antibodies against PEG were found not only in patients treated with PEGylated therapeutics, but also in $22 \%-25 \%$ of healthy blood donors, up from $0.2 \%$ two decades ago, which could be the consequence of the increasing use of PEG in cosmetics, therapeutics, and processed food. These preformed antibodies could affect the therapeutic efficacy of PEGylated drugs and impair their tolerance (4).

Recently, Schellenberger et al. have developed an alternative technology based on new unstructured, hydrophilic polypeptides, called XTEN (7), that can be fused to the proteins or peptides of interest and expressed recombinantly, providing in the same manner as PEG prolonged blood circulation, improved solubility, and stability of the fusion partner. Additionally, the XTEN technology allows convenient expression and purification procedures and other advantageous properties in comparison with PEG (Table 1). XTEN consists exclusively of the 6 amino acids A, E, G, P, S, and T and can serve as a purification tag. The use of XTEN can reduce the number of subsequent synthesis steps or even avoid them completely, for example, by combination with fluorescent proteins instead of chemical conjugation to fluorophores. Similar to PEG, XTEN binds a large hydrodynamic shell and forms an expanded conformation without fixed secondary or tertiary structures and is therefore heatresistant-a property that is partially transferred to the fused protein. In addition, the circulation half-life can be adapted by choosing exactly defined lengths, which also simplifies the characterization of 
TABLE 1

Comparison of PEGylation and Recombinant Polypeptide XTEN

\begin{tabular}{lc}
\multicolumn{1}{c}{ Properties } & PEGylation \\
\hline Large hydrate shell & + \\
Increases blood half-life & + \\
Increases stability & + \\
Can be used as tag for protein purification & + \\
Chemical coupling is needed & + \\
Site-directed PEGylation to cysteine & Heterogeneous \\
Size distribution & - \\
Biodegradable & - \\
\hline This table was partially adapted from Schellenberger et al. (7). & Cysteine-free for other labels \\
$+=$ Yes; $-=$ No. & + \\
\hline
\end{tabular}

the fusion products in contrast to the broad size distributions of PEGylated proteins. So far, XTEN peptide has been shown to be safe and nonimmunogenic in several animal species (7) and 2 XTEN-modified drugs have entered clinical phase I trials.

Annexin A5 (AnxA5) is a human protein $(35 \mathrm{kDa})$ that has been extensively used to detect phosphatidylserine-exposing cells (8), for example, for detection of apoptosis and cell death, which is altered in numerous diseases and treatments. Phosphatidylserines switch from the inner to the outer leaflet of the cell membrane within a few hours after initiating stimuli of apoptosis and other cell conditions and represent a highly accessible target for AnxA5 with a strong binding affinity (9). Many variants of human recombinant AnxA5 have been developed as probes for various imaging modalities, including MR imaging, optical imaging, ultrasound, and radionuclide-based imaging (10-15). However, because of its small size, wild-type AnxA5 is freely filtered by the kidneys and thus has a blood half-life of less than $7 \mathrm{~min}$ in mice (16). Therefore, target tissues with low perfusion rates might not achieve sufficient probe concentration for imaging.

Here, we report about the new imaging probe XTEN-AnxA5, a recombinant fusion protein containing human AnxA5 extended by a XTEN288 polypeptide, a variant of XTEN with 288 amino acids with an additional cysteine for coupling of thiol-reactive labels (Fig. 1).

\section{MATERIALS AND METHODS}

Complete methods and any associated references are available in the supplemental data (supplemental materials are available at http:// jnm.snmjournals.org).

In addition, the chemicals are discussed in the supplemental data.

\section{Expression and Purification of XTEN-AnxA5}

To design the fusion protein, an XTEN sequence of 288 amino acids (XTEN288) (7) was joined on to complementary DNA of the human AnxA5 gene (NP 1145.1). On the $\mathrm{N}$ terminus, 1 cysteine was added at the first position to allow subsequent labeling with thiol-reactive fluorophores or metal chelators for radioactive tracers. The endogenous cysteine of AnxA5 at position 316 was mutated to serine to avoid unspecific labeling (17). Two stop codons were added to eliminate C-terminal tag expression of the plasmid. Gene synthesis of XTEN alone and XTENAnxA5 fusion and the subcloning of these sequences into a pET30a(+) vector at NdeI and XhoI sites were performed by Genscript USA Inc.

Heat shock-competent Escherichia coli BL21(DE3) Gold Cells (Agilent Technologies) were transformed with the pET30(+) plasmid-carrying sequence of the XTEN or fusion protein and spread to grow on agar plates containing kanamycin $(100 \mu \mathrm{g} / \mathrm{mL})$. A single col- ony was picked to produce $10 \mathrm{~mL}$ of culture overnight in LB (LuriaBertani) medium with the same concentration of antibiotic growing at $30^{\circ} \mathrm{C}$. Further, the overnight culture was diluted 1:40 to inoculate $0.2 \mathrm{~L}$ of MagicMedia (Life Technologies) E. coli expression medium as a main culture. This culture was allowed to grow at $30^{\circ} \mathrm{C}, 300 \mathrm{rpm}$, for $7 \mathrm{~h}$ and was further cultivated at $23^{\circ} \mathrm{C}$ for $24 \mathrm{~h}$ by 300 -rpm shaking intensity.

Bacterial cells were then collected by centrifugation, and $8 \mathrm{~g}$ of wet cell pellet were lysed in $40 \mathrm{~mL}$ of BugBuster protein extraction reagent containing Benzonase endonuclease (Merck) at the recommended concentration and Proteinase Halt protease inhibitor cocktail

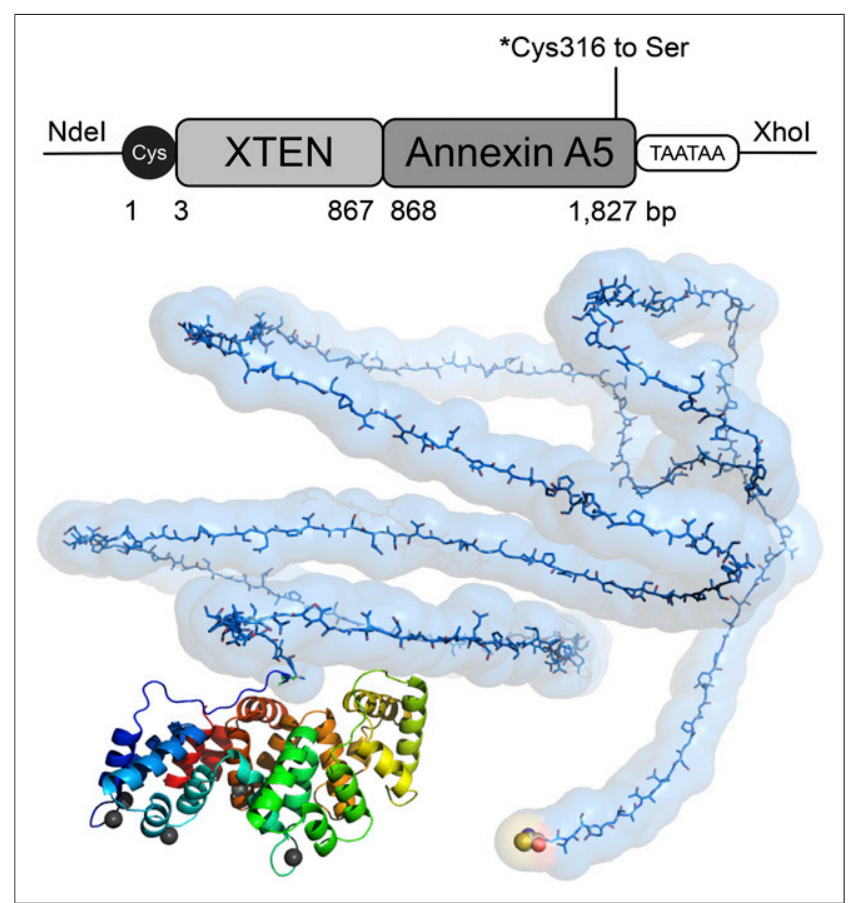

FIGURE 1. Models of XTEN-annexin A5. In schematic representation of DNA sequence, Cys indicates cysteine-codon for thiol-directed labeling reactions, ${ }^{*}$ Cys 316 to Ser is mutation site to avoid unwanted thiol reactions, and Ndel and Xhol are cloning sites. Molecular model of protein starts with cysteine at $\mathrm{N}$ terminus (colored spheres) for thioldirected chemical linkages, followed by hydrophilic unstructured polypeptide XTEN288 (blue). C terminus includes AnxA5 (colored), with calcium ions (gray) defining binding site to phosphatidylserine-exposing cell membranes. 


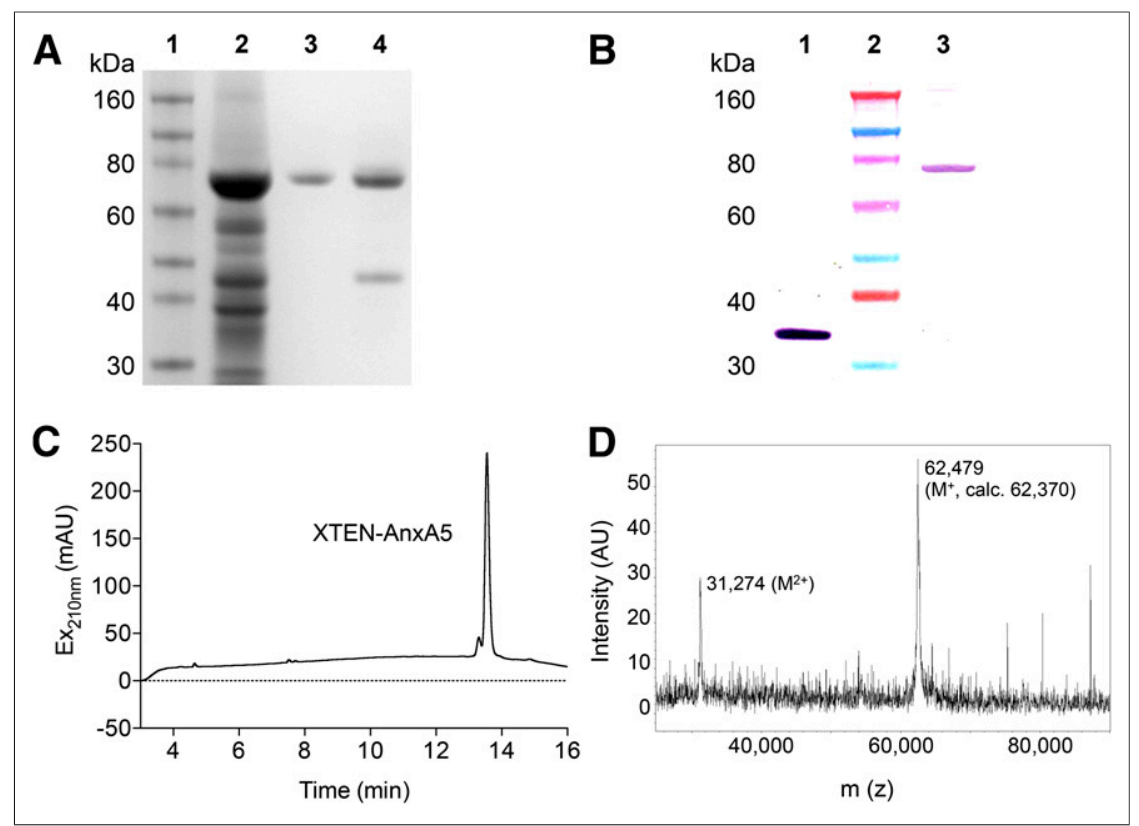

FIGURE 2. Nonreducing PAGE, Western blotting, RP-HPLC, and MALDI-TOF/MS of XTENAnxA5. (A) 4\%- to 12\%-gradient PAGE gel stained with Coomassie blue dye; lanes represent protein weight marker 1 , soluble fraction of $E$. coli lysate 2, products after first purification step using weak anion exchange column 4 , and second purification step using strong anion exchange column 3. (B) Western blotting with antibodies against AnxA5 of wild-type AnxA5 1 and purified XTEN-AnxA5 3, and protein weight marker 2. (C) RP-HPLC of purified XTEN-AnxA5 (retention time $=13.6 \mathrm{~min})$. (D) MALDI-TOF/MS of purified XTEN-AnXA5 $\left(\mathrm{M}^{+}\right.$and $\mathrm{M}^{2+}$ refer to singly and doubly charged ionic species of XTEN-AnXA5, respectively).

(Thermo Fisher). Bacterial cell lysate was loaded onto a 50-mL weak anion exchange column with diethylaminoethyl (DEAE) cellulose, equilibrated with starting buffer ( $20 \mathrm{mM}$ Tris, $50 \mathrm{mM} \mathrm{NaCl}, \mathrm{pH} 6.8$ ). The protein of interest was eluted with a gradient to end buffer $(20 \mathrm{mM}$ Tris, $0.5 \mathrm{M} \mathrm{NaCl}, \mathrm{pH} 6.8$ ) with a flow rate of $1 \mathrm{~mL} / \mathrm{min}$ using a BioLogic LP system (BioRad). The fractions containing the fusion protein were determined by sodium dodecyl sulfate polyacrylamide gel electrophoresis (SDS-PAGE) (Novex 4\%-12\% Bis-Tris gradient gel; Life Technologies) with subsequent Coomassie Simply Blue SafeStain (Life Technologies) and then pooled, and the elution buffer was exchanged to the equilibration buffer (20 mM Tris, $50 \mathrm{mM} \mathrm{NaCl}, \mathrm{pH}$ 6.8). The solution was loaded onto a 50-mL strong anion exchange column with UnosphereQ (BioRad) (equilibrated with $20 \mathrm{mM}$ Tris, $50 \mathrm{mM} \mathrm{NaCl}, \mathrm{pH}$ 6.8), and the protein of interest was again eluted using a gradient to end buffer (20 mM Tris, 1 $\mathrm{M} \mathrm{NaCl}, \mathrm{pH}$ 6.8) with a flow rate of $1 \mathrm{~mL} / \mathrm{min}$. XTEN-AnxA5 fractions were selected as described above and then pooled, and the concentration of $\mathrm{NaCl}$ in the buffer was increased to $3 \mathrm{M}$. This solution was added to a 30-mL Octyl-Sepharose 4 Fast Flow (Sigma-Aldrich) hydrophobic interaction column, which was equilibrated with high salt buffer $(20 \mathrm{mM}$ Tris, $3 \mathrm{M} \mathrm{NaCl}, \mathrm{pH}$ 7.5), and the desired protein was eluted using a decreasing gradient to end buffer (20 mM Tris, $135 \mathrm{mM} \mathrm{NaCl}, \mathrm{pH} 7.5$ ). XTEN-AnxA5 fractions were selected again as described above, then pooled, and desalted against storing buffer (10 mM HEPES [(4-(2hydroxyethyl)-1-piperazineethanesulfonic acid)], $135 \mathrm{mM} \mathrm{NaCl}, \mathrm{pH}$ 7.5). XTEN288 was expressed, purified, and analyzed as described by Schellenberger et al. (7) (sequence in supplemental data). The protein solution was passed through $0.22-\mu \mathrm{m}$ filter devices, and concentrations of XTEN and XTEN-AnxA5 were measured using a bicinchoninic acid protein assay.

Because XTEN lacks the amino acids tryptophan and tyrosine, which are mainly responsible for the colorimetric reaction of the BCA assay, a correction factor for XTEN and XTEN-AnxA5 protein concentration measurements in comparison to the albumin standard was calculated. The determination of the factor of the XTEN polypeptide concentration, measured using a BCA assay, in comparison with the weight of lyophilized XTEN polypeptide revealed a correction factor of 4.0 for XTEN288 and 1.4 for XTEN-AnxA5.

Western blotting analysis, high-performance liquid chromatography (HPLC), and MALDITOF/MS (matrix-assisted laser desorption/ionization time-of-flight mass spectrometry) are described in the supplemental data.

\section{Coupling of XTEN-AnxA5 with Diethylene Triamine Pentaacetic Acid (DTPA) and 6S-IDCC}

Before labeling, 1,100 $\mu \mathrm{L}$ of XTEN-AnxA5 with a concentration of $1.8 \mathrm{mg} / \mathrm{mL}$ was treated with dithiothreitol (DTT) (10 mM final concentration) to reduce potential cysteine dimers at the $\mathrm{N}$ terminus. XTEN-AnxA5 was therefore incubated with DTT for $90 \mathrm{~min}$ at $37^{\circ} \mathrm{C}$. To remove DTT, the solution of reduced XTENAnxA5 was dialyzed for $24 \mathrm{~h}$ at $4^{\circ} \mathrm{C}$ in buffer containing $10 \mathrm{mM}$ HEPES, $140 \mathrm{mM}$ $\mathrm{NaCl}$, and $1 \mathrm{mM}$ ethylenediaminetetraacetic acid (EDTA), pH 7.4. Maleimide-DTPA or the near-infrared fluorescence (NIRF) dye maleimide-6S-IDCC (Em Max $_{\text {[maximal emis- }}$ sion $]=695 \mathrm{~nm}, \mathrm{Ex}_{\mathrm{Max}}$ [maximal excitation] $=675 \mathrm{~nm}$ ) was dissolved in water at a concentration of $10 \mathrm{~g} / \mathrm{L}$ and then added to the protein solution in a 20 -fold molar excess. After a 24 -h reaction time at $4{ }^{\circ} \mathrm{C}$, unbound maleimide-DTPA or maleimide-6S-IDCC was removed by 2 rounds of gel filtration using centrifugal spin columns with BioGel P6. The labeling of AnxA5 was done accordingly (6S-IDCCAnxA5). BioGel (BioRad) P6 (10 mL) was equilibrated with $10 \mathrm{mM}$ HEPES, $140 \mathrm{mM} \mathrm{NaCl}, \mathrm{pH}$ 7.4; centrifugation steps included $3 \mathrm{~min}$ (first round) and $5 \mathrm{~min}$ (second round) at $1,500 \mathrm{~g}$. The final protein concentration was determined by BCA protein assay. The labeling ratio of protein with maleimide-6S-IDCC was calculated after measuring the dye absorption at $675 \mathrm{~nm}\left(\varepsilon=240,000 \mathrm{M}^{-1} \mathrm{~cm}^{-1}\right)$. The labeling ratio of the maleimide-DTPA was determined using complexation of $\mathrm{Eu}^{3+}$. Unbound $\mathrm{Eu}^{3+}$ was removed using centrifugal spin columns, and the concentration of remaining $\mathrm{Eu}^{3+}$ was calculated after measuring the fluorescence of the europium chelate formed using an enhancer solution (18).

\section{Labeling of XTEN-Anx-DTPA with ${ }^{111}$ nn $^{3+}$}

XTEN-AnxA5-DTPA $(121.2 \mu \mathrm{g}, 52 \mu \mathrm{L})$ was mixed with $463 \mu \mathrm{L}$ of buffer (270 mM NaOAc/HOAc, $79 \mathrm{mM}$ Gentisin, pH 5.0). The resulting solution was added to $500 \mu \mathrm{L}$ of ${ }^{111} \mathrm{InCl}_{3}$ solution $(0.02 \mathrm{M} \mathrm{HCl}$, $291 \mathrm{MBq}, 157.3 \mathrm{ng}$ of indium), thoroughly mixed, and incubated for $70 \mathrm{~min}$ at room temperature. Thereafter, the labeled protein was purified by 3 rounds of ultrafiltration with $10-\mathrm{kDa}$ Amicon Ultra 0.5 Centrifugal Filters (Millipore) by adding $1 \mathrm{~mL}$ of buffer $(10 \mathrm{mM}$ HEPES, $140 \mathrm{mM} \mathrm{NaCl}$, pH 7.4) each time. The radioactivity of both column and filtrate was measured after each step. The procedure yielded $206 \mathrm{MBq}$ of XTEN-AnxA5- ${ }^{111} \mathrm{In}$ in $160 \mu \mathrm{L}$ of buffer solution ( $71 \%$ yield related to the used ${ }^{111} \mathrm{In}^{3+}$ ).

Flow cytometry and fluorescence microscopy imaging; calcium titration curves of binding to aged red blood cells (RBC); and SPECT/ CT, NIRF imaging, and immunohistology are described in the supplemental data. 


\section{Molecular Modeling}

The molecular model of XTEN-AnxA5 was done using Pymol (19) software and Marvin (ChemAxon Software) based on the sequence of the unstructured XTEN288 (7) and the crystal structure of AnxA5 (20).

\section{RESULTS}

\section{Expression and Purification of XTEN-AnxA5 Fusion Protein}

The designed gene sequence (Fig. 1; supplemental data) was subcloned into a pET vector and expressed in E. coli using an adapted protocol (described in the "Materials and Methods" section), resulting in high expression of XTEN-AnxA5 (Fig. 2A, whole-cell lysate). The calculated molecular weight of XTEN-AnxA5 is $63 \mathrm{kDa}$, but it migrates more slowly in SDS-PAGE (apparent molecular weight of $73 \mathrm{kDa}$ ), which is typical for XTEN fusion proteins (7) due to the large hydrate shell of XTEN.

Because of its high content of negatively charged amino acids, the unstructured chain of XTEN is highly suited to be used as a tag for recombinant protein purification. The wholecell lysate, containing XTEN-AnxA5, was purified using weak and strong anionic exchange columns equilibrated with low-salt buffer, and the protein was eluted using a gradient with increasing salt concentration. Two purification steps were sufficient to remove host cell proteins, DNA, and possible aggregates. After these 2 columns, there remained fragments of XTEN-AnxA5, barely visible in Coomassie-stained SDS-PAGE gel, that were identified via reversed-phase HPLC and had the same retention time $(5.1 \mathrm{~min})$ as XTEN288, expressed and purified under similar conditions (Supplemental Fig. 1). To remove these incomplete XTEN fragments that did not contain AnxA5, a third purification step with a hydrophobic interaction column was performed. A sterile solution of XTEN-AnxA5 in storing buffer, showing good purity as a single band on a nonreducing SDS-PAGE gel (Fig. 2A), was used for successive labeling procedures. After treatment with dithiothreitol to reduce XTEN-AnxA5-thiol dimers and after all purification steps, an HPLC analysis was done that showed a purity of 95\% (Fig. 2C). The identity was further confirmed by mass spectroscopy, giving a mass of $62.48 \mathrm{kDa}$, which for this size protein is within the typical range of the calculated mass of $62.37 \mathrm{kDa}$ (Fig. 2D). After the expression and purification process, the final protein yield was about $9 \mathrm{mg}$ by BCA from $0.2 \mathrm{~L}$ bacterial culture. The necessary BCA correction factor with 1.4 for XTEN-AnxA5 and 4.0 for XTEN was determined by weighing in lyophilized proteins as described in the "Materials and Methods" section. Purified XTEN-AnxA5 was further characterized by Western blotting (Fig. 2B), showing that antibodies directed to wild-type AnxA5 labeled the fusion protein as well. The band of XTEN-AnxA5 is less intense than that of wild-type AnxA5, which can be attributed to reduced binding of the antibody or reduced transfer to the nitrocellulose membrane during Western blotting due to the XTEN hydrate shell, as described for a Factor VII-XTEN fusion protein (7).

\section{Labeling of XTEN-AnXA5}

XTEN-AnxA5 was successfully coupled and purified with either maleimide-DTPA for labeling with nuclear tracers or with the NIRF dye maleimide-6S-IDCC for optical imaging. The labeling efficiency of the resulting DTPA-XTEN-AnxA5 and 6S-IDCC-XTEN-AnxA5 was $60 \%$ and $61 \%$, respectively.

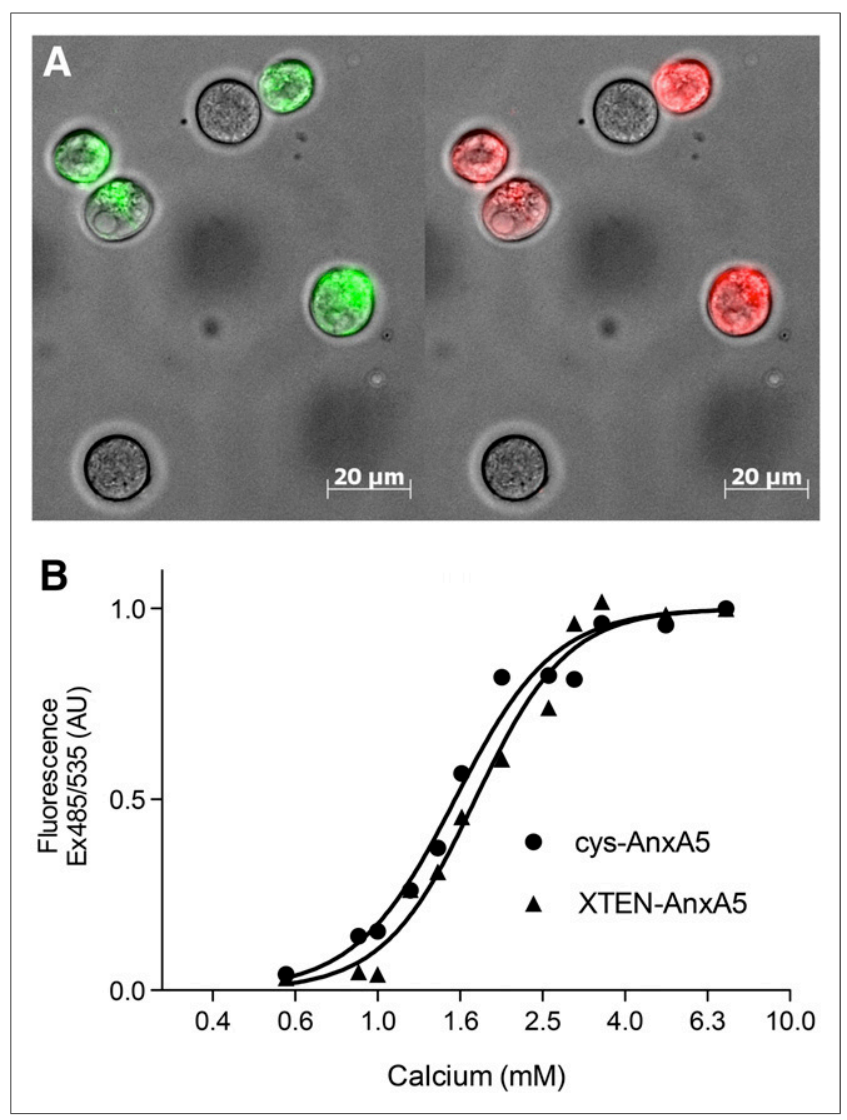

FIGURE 3. Characterization of binding capabilities of XTEN-AnxA5 fusion protein to apoptotic cells. (A) Fluorescent microscopy of camptothecin-treated Jurkat $T$ cells double-stained with fluorescein-labeled wild-type AnxA5 (green) and 6S-IDCC-labeled XTEN-AnxA5 fusion protein (red). Apoptotic cells are positive for both annexins, and nonapoptotic cells are negative for both. (B) Calcium titration curves of binding of FITC-labeled wt-AnxA5 (wild-type) in contrast to site-directed fluorescein-maleimide-labeled cys-AnxA5 and XTEN-AnxA5 to phosphatidylserine-expressing $\mathrm{RBC}$. $A U$ = arbitrary units.

\section{Binding of XTEN-AnxA5 Fusion Protein to Phosphatidylserine-Presenting Cells}

Camptothecin-treated Jurkat T cells with $49 \%$ apoptotic/dead cells and control cells with $2 \%$ apoptotic/dead cells were incubated with fluorescein isothiocyanate (FITC)-labeled wild-type AnxA5 and with 6S-IDCC-XTEN-AnxA5 as a 1:1 mixture (Fig. 3A). Fluorescence microscopy images of the cells directly after incubation revealed that 6S-IDCC-XTEN-AnxA5 (red) selectively binds to apoptotic/dead cells in a manner similar to wild-type FITC-AnxA5 (green). Flow cytometry analysis of both cell groups confirmed these results (Supplemental Fig. 2).

To assess the calcium-dependent binding affinity of XTENAnxA5, we performed a calcium titration assay on phosphatidylserine-presenting aged RBC as described by Tait et al. (21) and compared it with AnxA5, a mutant of wild-type AnxA5, that has, like XTEN-AnxA5, an additional cysteine for site-directed covalent labeling. The calcium-dependent half-maximal binding of XTEN-AnxA5 as shown in Figure 3B was at $1.73 \mathrm{mM} \mathrm{CaCl} 2$ (95\% confidence interval, 1.62-1.83, hill slope 3.9), compared with $1.54 \mathrm{mM} \mathrm{CaCl}_{2}$ for AnxA5 (95\% confidence interval, 1.45-1.64, hill slope 3.6). 


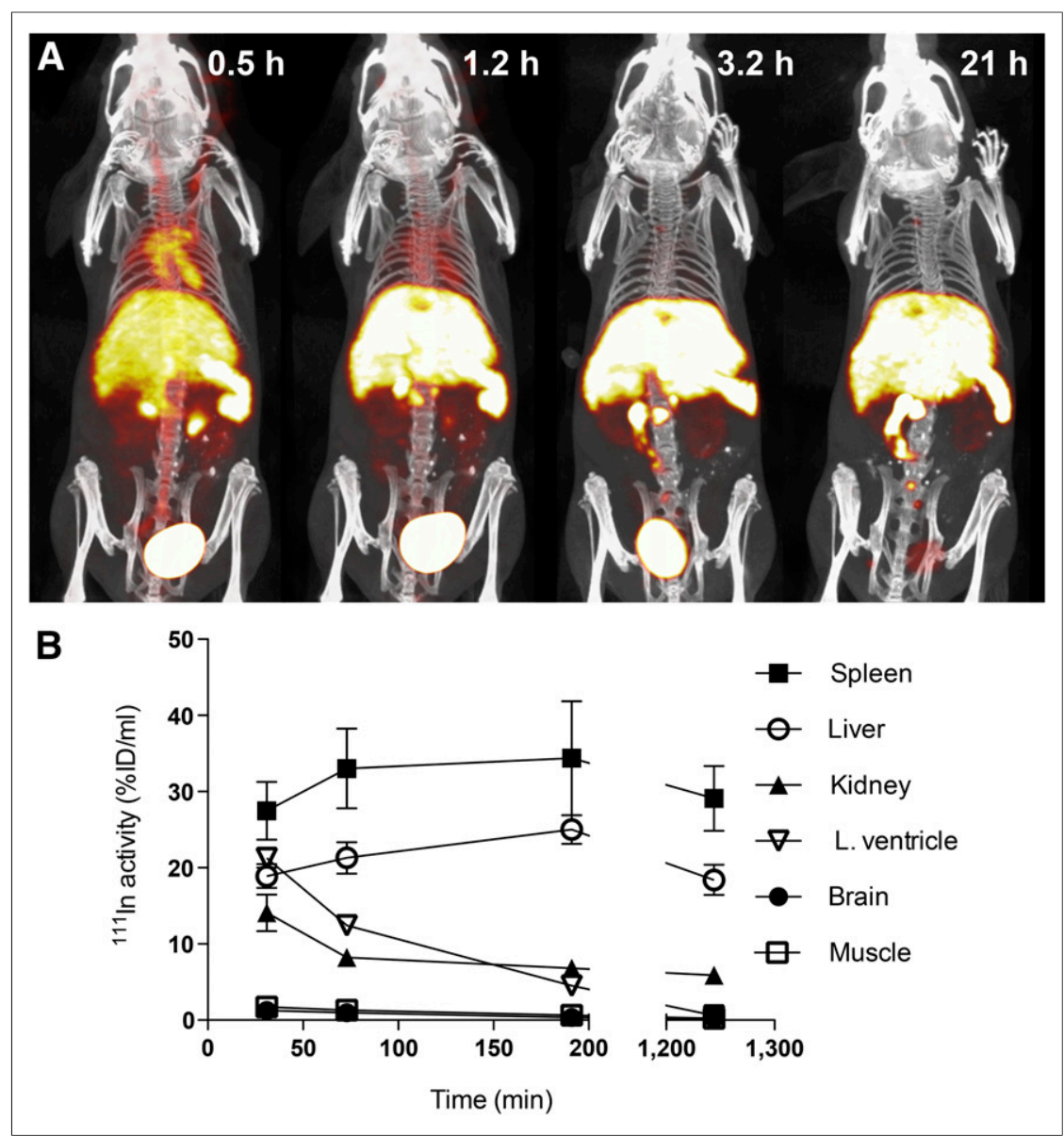

FIGURE 4. SPECT/CT of ${ }^{111}$ In-labeled DTPA-XTEN-AnXA5 for determination of blood half-life. (A) Maximal-intensity projections of mouse after injection of ${ }^{111}$ In-labeled XTEN-AnXA5 are shown for 4 time points after injection. Blood half-life was determined to be $63 \min (n=5)$. (B) Time course of in vivo distribution in several organs (mean, SEM).

\section{Prolonged Circulation Time and Higher Tumor Uptake of XTEN-AnxA5 Under Chemotherapy}

DTPA-XTEN-AnxA5, labeled with radioactive ${ }^{111}$ In, was injected intravenously and circulated for about $3 \mathrm{~h}$ in the vascular system of the mice (Fig. 4). The blood half-life was calculated to be $63.3 \pm 4.4 \mathrm{~min}(n=5)$ from regions of interest located in the left cardiac ventricle of the SPECT/CT images. Figure 4 shows the in vivo time course of the tracer up to $1 \mathrm{~d}$. The fusion protein was cleared from blood by the spleen, liver, and kidneys.

The ex vivo biodistribution $1 \mathrm{~d}$ after injection confirmed these results (Fig. 5). The highest indium-specific activity per gram of tissue was detected in the spleen, liver, and kidneys. Unspecific accumulation in other organs was low but was slightly elevated in the gastrointestinal tract, which could be due to a naturally higher cell turnover and apoptosis of the gut epithelium and lymphatic system.

To test whether the prolonged blood half-life of XTEN-AnxA5 could improve target accumulation, we implanted tumor xenografts (EL4 cell line) into the flanks of immunodeficient nude mice and treated them with etoposide and cyclophosphamide to induce tumor apoptosis. The mice were injected intravenously the next day with the NIRF-labeled probes 6S-IDCC-XTEN-AnxA5 or 6SIDCC-AnxA5. Three hours after injection, the tumors were imaged ex vivo together with other organs (Fig. 6). 6S-IDCC-
XTEN-AnxA5 demonstrated a significantly higher tumor uptake than 6S-IDCCAnxA5, with respective values of 2.37 vs. 0.986 arbitrary units $(n=4$ each, $P=$ 0.03 ), which was even more pronounced for tumor hot spot areas, with values of 3.80 vs. 1.36 arbitrary units $(n=4$ each, $P=0.03)$. Ex vivo fluorescence microscopy of the tumors demonstrated a higher uptake for the long-circulating 6S-IDCCXTEN-AnxA5 accordingly. To confirm that all tumors had similar levels of apoptosis, immunohistology using antibodies against activated caspase- 3 was performed on fixed frozen sections of all tumor explants, with representative images shown in Figure 6C.

\section{DISCUSSION}

The motivation for the present study was to demonstrate that the short blood circulation time of AnxA5 as a typical small protein-based imaging probe can be extended by recombinant expression with $\mathrm{XTEN}$ as an alternative to PEGylation (22). We successfully adapted the method of Schellenberger et al. (7) to design an XTEN-AnxA5 fusion protein with a cysteine coupling site and demonstrated its biologic activity by flow cytometry and fluorescence microscopy in vitro and its extended blood half-life of $1 \mathrm{~h}$, compared with unmodified AnxA5, by nuclear imaging. Similar to PEGylated AnxA5 (22), XTEN-AnxA5 showed a reduced blood clearance by the kidneys and an increased blood clearance by the spleen and liver. The renal excretion should decrease further, if longer XTEN variants would be attached to the protein (7). Moreover, we could demonstrate that this longer circulation time allows a higher accumulation in chemotherapy-treated tumors of fluorescently labeled XTEN-AnxA5 in comparison to similarly labeled wild-type AnxA5, which could be partly due to a target-independent enhanced permeability and retention effect elicited by the increased size of XTEN-AnxA5.

After chemical lysis of the E. coli culture, the lysate was purified by 2 successive anion exchange columns. This is possible because XTEN has a high content of negatively charged glutamic acid residues (17\% of amino acids) and can therefore readily serve as a powerful purification tag without the potential disadvantages of other tags such as increased immunogenicity or necessity of enzymatic removal.

Not removed by these columns were fractions of XTENAnxA5, which behaved similarly to XTEN288 without AnxA5 fragments by comparison with independently expressed XTEN288 (Supplemental Fig. 1). XTEN alone is virtually not detectable in Coomassie-stained protein gels and is underdetected by a factor of 4.0 in BCA assays, compared with albumin (see above). XTEN288 is more visible in silver-stained protein gels and can be detected in HPLC at $210 \mathrm{~nm}$. These XTEN-like fragments could be removed by a hydrophobic interaction column. This 3- 


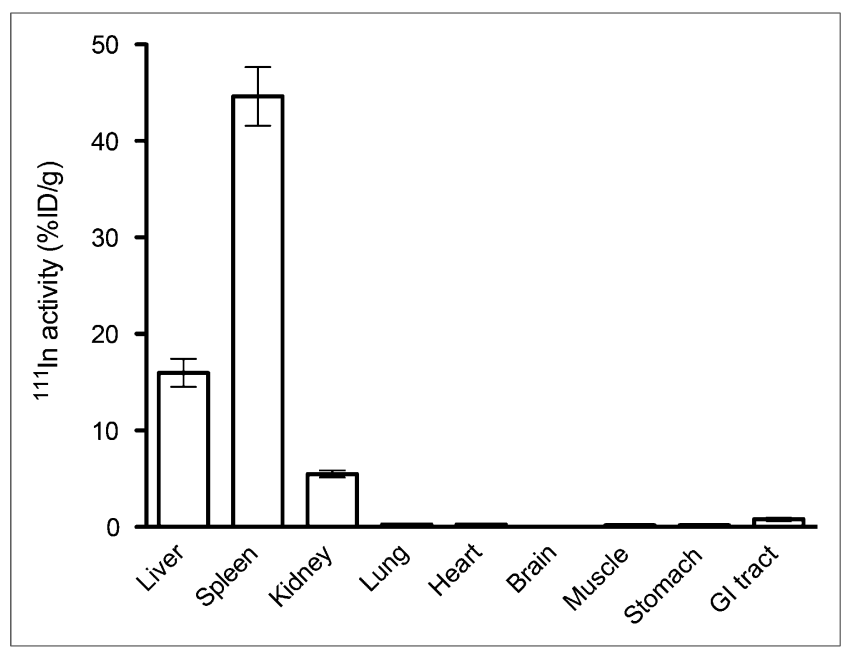

FIGURE 5. Ex vivo tissue activity of ${ }^{111} \mathrm{In}$-DTPA-AnXA5-XTEN $1 \mathrm{~d}$ after injection. XTEN-AnXA5 that was not excreted by kidneys mainly accumulated in liver and spleen. Unspecific uptake in other organs was minimal. Slightly higher uptake in gastrointestinal (GI) tract might be due to high cell turnover.

step chromatographic separation resulted in good purity of the final product as determined by HPLC and MALDI-TOF/MS (Fig. 2).

The selectivity of XTEN-AnxA5 to apoptotic cells was comparable to commercial AnxA5-FITC, as demonstrated by fluorescence microscopy (Fig. 3A) and flow cytometry (Supplemental Fig. 2). The calcium-dependent binding affinity to phosphatidylserine-presenting aged RBC (21) of XTEN-AnxA5 was similar to AnxA5 (Fig. 3B), confirming good binding properties of XTEN-AnxA5. Both proteins were coupled site-directed with fluorescein maleimide at the singular cysteines.

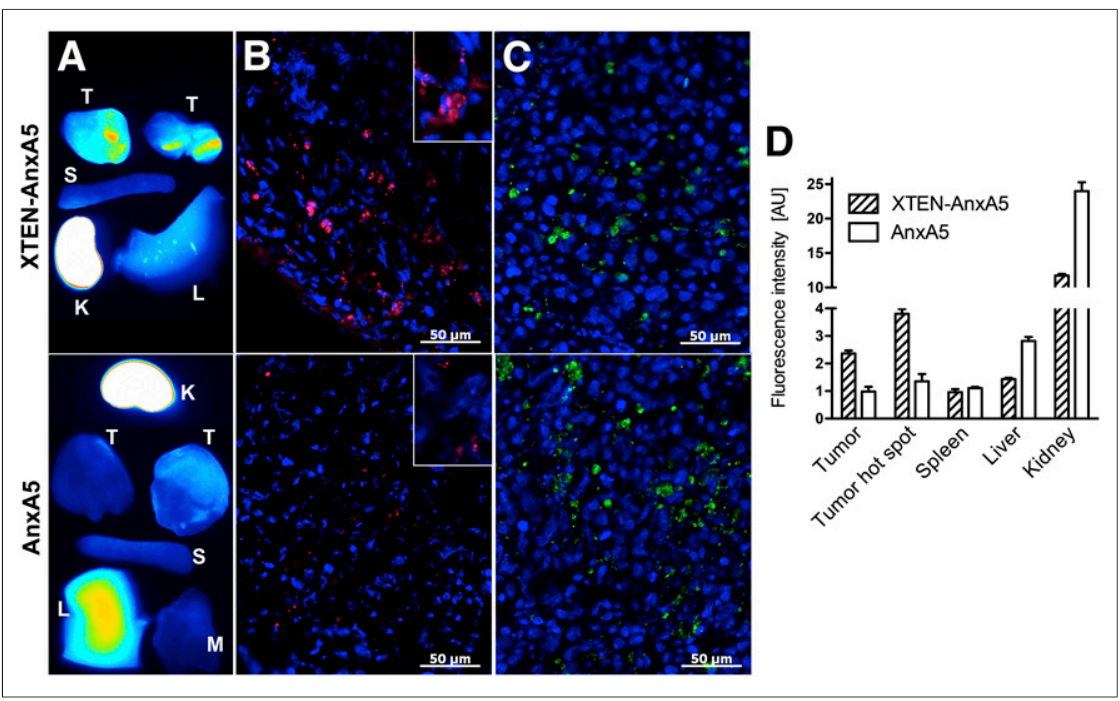

FIGURE 6. Comparison of NIRF imaging with XTEN-AnxA5 (upper) and AnxA5 (lower). (A) EX vivo NIRF imaging with 6S-IDCC-XTEN-AnXA5 and 6S-IDCC-AnXA5 $3 \mathrm{~h}$ after intravenous injection in tumor-bearing mice under chemotherapy. (B) Ex vivo NIRF microscopy confirmed higher uptake of XTEN-AnxA5 than AnxA5. (C) Both tumor groups ( $n=4$ each) had similar areas of high apoptosis as shown by immunohistology. Cell nuclei stained with Hoechst 33258 (blue), 6S-IDCC (red), and activated caspase-3 (green). (D) Quantification of NIRF imaging revealed higher tumor uptake for XTEN-AnxA5, especially in hot spot areas. $\mathrm{K}=$ kidney; $\mathrm{L}=$ liver; $\mathrm{M}=$ muscle; $\mathrm{S}=$ spleen; $\mathrm{T}=$ tumor. $\mathrm{AU}=$ arbitrary units.
The blood half-life of $1 \mathrm{~h}$ in mice was within an expected range when compared with other XTEN-fusion proteins (7) and markedly longer than that of wild-type AnxA5. If required, it could be further extended by fusing longer variants of XTEN (XTEN576 or XTEN864) or by tandem dimerization of the XTEN-AnxA5 construct. These constructs could be useful to optimize the circulation time or to perform AnxA5 imaging under near-steadystate conditions. Longer variants should have a further reduced kidney clearance and hence increased clearance by liver and spleen.

By using XTEN we were able to avoid several important disadvantages of PEG (Table 1), among which safety and costs are especially relevant with respect to clinical applications. First, PEGylation adds a synthesis and purification step whereas XTEN simplifies purification by serving as a tag. Second, PEGylation can result in partial inactivation of the target protein, especially when ng amine-directed coupling (22), a complication to which AnxA5 is especially susceptible (23). Third, PEGylation often results in mixtures of proteins with zero-, one-, and multiple-coupled PEGs, which are hard to separate (22), in addition to the fact that PEG is usually provided as a mixture of heterogeneous lengths (24). In contrast, XTEN-coupled products are developed by genetic fusion to a defined amino acid sequence that ensures a monodisperse final compound (Figs. 2C and 2D) without inactive variants or free polypeptides, avoiding labor and potentially cost-intensive coupling and purification procedures, compared with PEGylation. This is especially important if a clinical application is intended. The fact that the product is a single species, in contrast to the wide mass distribution of PEGylated products, facilitates characterization of the product itself or modifications by techniques such as MALDImass analysis, as demonstrated. Moreover, on the basis of a mutated AnxA5 variant incapable of binding to phosphatidylserineexposing cells (25), an inactive control probe could be designed with virtually the same mass, structure, and therefore blood halflife to correct for biodistribution by simultaneous application with XTEN-AnxA5.

Last, there are increasing safety concerns about PEGylation. Recently, it has been reported that PEGylated protein complexes may dissociate in vivo, resulting in free PEG moieties circulating in the bloodstream and tissues (5). This finding raises questions about potential long-term toxicity because PEG is nonbiodegradable (26). In contrast, the XTEN sequence consists of natural amino acids and should be efficiently biodegraded like any other protein (7). As mentioned in the introduction, there are new reports about circulating antibodies against PEG in animals and patients treated with PEGylated compounds. Special attention should be given to a recent report showing evidence that in about $25 \%$ of healthy blood donors antibodies against PEG can be detected, most likely induced by the use of PEG-containing cosmetics, drugs, or processed food. Preexisting antibodies could induce faster degradation and clearance of therapeutics from the blood, thereby lowering the efficacy of PEGylated products (4). Some reports indicate that 
significant accumulation PEGylated protein, occurring with longterm treatment at high dosages, may increase the risk of liver toxicity (2). XTEN showed a low immunogenicity in animal models, even in the presence of adjuvant (7). The risk of cross-reactivity or autoimmune response appears to be minimal because the XTEN sequence does not have any homology to known human endogenous proteins $(7,27,28)$.

In summary, long-circulating XTEN-AnxA5 was successfully expressed and purified with high yield to improve the detection of apoptotic/dead cells in vivo. The $\mathrm{N}$-terminal cysteine could be effectively used for site-specific labeling with a thiol-reactive NIRF dye for optical imaging or a DTPA chelator for nuclear imaging. The fusion protein showed equivalent functionality for binding to apoptotic cells as compared with AnxA5 in vitro, whereas the circulation time in the blood was markedly increased to about $1 \mathrm{~h}$, which could be optimal for many applications. In vivo, this resulted in an increased probe accumulation in tumors, compared with AnxA5. The advantages of XTEN-AnxA5 need to be confirmed in future more comprehensive animal studies.

\section{CONCLUSION}

This new method of half-life extension demonstrates several important advantages over PEGylation, whose conception as a completely safe technique is recently being called into question. Diagnostic agents in particular require the highest safety standards, because of their application to potentially healthy people, leading us to believe that XTEN fusion is a promising alternative for the design of long-circulating protein- or peptide-based imaging probes.

\section{DISCLOSURE}

The costs of publication of this article were defrayed in part by the payment of page charges. Therefore, and solely to indicate this fact, this article is hereby marked "advertisement" in accordance with 18 USC section 1734. The work was supported by the Deutsche Forschungsgemeinschaft DFG (SCH 1416/2-2, SCH 1416/ $3-2)$. No other potential conflict of interest relevant to this article was reported.

\section{ACKNOWLEDGMENTS}

We thank Gesche Genter for assisting and performing various experiments and Randall Lindquist for language editing.

\section{REFERENCES}

1. Kang JS, Deluca PP, Lee KC. Emerging PEGylated drugs. Expert Opin Emerg Drugs. 2009;14:363-380.

2. Jevsevar S, Kunstelj M, Porekar VG. PEGylation of therapeutic proteins. Biotechnol J. 2010;5:113-128.

3. Bendele A, Seely J, Richey C, Sennello G, Shopp G. Short communication: renal tubular vacuolation in animals treated with polyethylene-glycol-conjugated proteins. Toxicol Sci. 1998;42:152-157.

4. Garay RP, El-Gewely R, Armstrong JK, Garratty G, Richette P. Antibodies against polyethylene glycol in healthy subjects and in patients treated with PEG-conjugated agents. Expert Opin Drug Deliv. 2012;9:1319-1323.
5. Elliott VL, Edge GT, Phelan MM, et al. Evidence for metabolic cleavage of a PEGylated protein in vivo using multiple analytical methodologies. Mol Pharm. 2012;9:1291-1301.

6. Suzuki T, Ichihara M, Hyodo K, et al. Accelerated blood clearance of PEGylated liposomes containing doxorubicin upon repeated administration to dogs. Int J Pharm. 2012;436:636-643.

7. Schellenberger V, Wang C-W, Geething NC, et al. A recombinant polypeptide extends the in vivo half-life of peptides and proteins in a tunable manner. Nat Biotechnol. 2009;27:1186-1190.

8. Zhang C, Feng Y, Qu S, et al. Resveratrol attenuates doxorubicin-induced cardiomyocyte apoptosis in mice through SIRT1-mediated deacetylation of p53. Cardiovasc Res. 2011;90:538-545.

9. Galluzzi L, Vitale I, Abrams JM, et al. Molecular definitions of cell death subroutines: recommendations of the Nomenclature Committee on Cell Death 2012. Cell Death Differ. 2012;19:107-120.

10. Schellenberger EA, Bogdanov A, Petrovsky A, Ntziachristos V, Weissleder R, Josephson L. Optical imaging of apoptosis as a biomarker of tumor response to chemotherapy. Neoplasia. 2003;5:187-192.

11. Ntziachristos V, Schellenberger EA, Ripoll J, et al. Visualization of antitumor treatment by means of fluorescence molecular tomography with an annexin V-Cy5.5 conjugate. Proc Natl Acad Sci USA. 2004;101:12294-12299.

12. Sosnovik DE, Schellenberger EA, Nahrendorf M, et al. Magnetic resonance imaging of cardiomyocyte apoptosis with a novel magneto-optical nanoparticle. Magn Reson Med. 2005;54:718-724.

13. Bahmani P, Schellenberger E, Klohs J, et al. Visualization of cell death in mice with focal cerebral ischemia using fluorescent annexin A5, propidium iodide, and TUNEL staining. J Cereb Blood Flow Metab. 2011;31:1311-1320.

14. Hofstra L, Dumont EA, Thimister PW, et al. In vivo detection of apoptosis in an intracardiac tumor. JAMA. 2001;285:1841-1842.

15. Kartachova M, Haas RL, Olmos RA, Hoebers FJ, van Zandwijk N, Verheij M. In vivo imaging of apoptosis by ${ }^{99 \mathrm{~m}} \mathrm{Tc}$-annexin $\mathrm{V}$ scintigraphy: visual analysis in relation to treatment response. Radiother Oncol. 2004;72:333-339.

16. Blankenberg FG, Tait J, Ohtsuki K, Strauss HW. Apoptosis: the importance of nuclear medicine. Nucl Med Commun. 2000;21:241-250.

17. Tait JF, Brown DS, Gibson DF, Blankenberg FG, Strauss HW. Development and characterization of annexin $\mathrm{V}$ mutants with endogenous chelation sites for ${ }^{99 \mathrm{~m}}$ Tc. Bioconjug Chem. 2000;11:918-925.

18. Hemmilä I, Dakubu S, Mukkala VM, Siitari H, Lövgren T. Europium as a label in time-resolved immunofluorometric assays. Anal Biochem. 1984;137:335-343.

19. DeLano WL. The PyMOL Molecular Graphics System. New York, NY: DeLano Scientific, USA; 2002.

20. Huber R, Römisch J, Paques EP. The crystal and molecular structure of human annexin $\mathrm{V}$, an anticoagulant protein that binds to calcium and membranes. EMBO J. 1990;9:3867-3874.

21. Tait JF, Gibson DF, Smith C. Measurement of the affinity and cooperativity of annexin V-membrane binding under conditions of low membrane occupancy. Anal Biochem. 2004;329:112-119.

22. Wen X, Wu Q-P, Ke S, et al. Improved radiolabeling of PEGylated protein: PEGylated annexin V for noninvasive imaging of tumor apoptosis. Cancer Biother Radiopharm. 2003;18:819-827.

23. Schellenberger EA, Sosnovik D, Weissleder R, Josephson L. Magneto/optical annexin V, a multimodal protein. Bioconjug Chem. 2004;15:1062-1067.

24. Huang Z, Zhu G, Sun C, et al. A novel solid-phase site-specific PEGylation enhances the in vitro and in vivo biostabilty of recombinant human keratinocyte growth factor 1. PLoS ONE. 2012;7:e36423.

25. Mira JP, Dubois T, Oudinet JP, Lukowski S, Russo-Marie F, Geny B. Inhibition of cytosolic phospholipase A2 by annexin V in differentiated permeabilized HL60 cells: evidence of crucial importance of domain I type II Ca2+-binding site in the mechanism of inhibition. J Biol Chem. 1997;272:10474-10482.

26. Chen C, Constantinou A, Deonarain M. Modulating antibody pharmacokinetics using hydrophilic polymers. Expert Opin Drug Deliv. 2011;8:1221-1236.

27. Cleland JL, Geething NC, Moore JA, et al. A novel long-acting human growth hormone fusion protein (VRS-317): enhanced in vivo potency and half-life. J Pharm Sci. 2012;101:2744-2754.

28. Geething NC, To W, Spink BJ, et al. Gcg-XTEN: an improved glucagon capable of preventing hypoglycemia without increasing baseline blood glucose. PLoS ONE. 2010;5:e10175. 